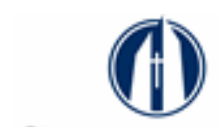

George Fox

UNIVERSITY

Digital Commons @ George Fox University

Faculty Publications - Grad School of Clinical

Psychology

Graduate School of Clinical Psychology

2016

Perceptions of Caregiver Distress, Health Behaviors, and Provider Health-Promoting Communication and their Relationship to Stress Management in MS Caregivers

Lauren Penwell-Waines

Marie-Christine Goodworth

Rhonda S. Casillas

Lara Stepleman

Follow this and additional works at: https://digitalcommons.georgefox.edu/gscp_fac

Part of the Psychology Commons 


\title{
Perceptions of caregiver distress, health behaviors, and provider health-promoting communication and their relationship to stress management in MS caregivers
}

\author{
Lauren Penwell-Waines ${ }^{a}$, Marie-Christine Rutter Goodworth ${ }^{b}$, Rhonda S. Casillasc, Rebecca Rahn ${ }^{d}$ and Lara Stepleman ${ }^{a}$ \\ aDepartment of Psychiatry and Health Behavior, Medical College of Georgia at Georgia Regents University; ${ }^{b}$ Graduate Department of Clinical \\ Psychology, George Fox University; 'Arizona State University Counseling Services; ${ }^{d}$ Augusta MS Center
}

\begin{abstract}
This study applied the Stress/Health Model to examine a novel approach for promoting stress management among 67 caregivers of persons with multiple sclerosis, who often face unique caregiving challenges. Hierarchical regressions indicated that caregiver distress (i.e., emotional burden) and engagement in other health-promoting activities (i.e., controlling alcohol use) were the best predictors of caregiver stress management. Communication with the MS care recipient's health provider about caregiver engagement in health-promoting activities was associated with caregiver stress management, but not significantly more so than explained by the other factors (i.e., caregiver distress and engagement in health-promoting behaviors). A more controlled study would be indicated to further explain how to encourage, within the medical setting, caregiver engagement in self-care activities.
\end{abstract}

Caring for persons with multiple sclerosis (PwMS) can be a difficult and demanding role, given the variable course of the disease and the multifaceted nature of the symptom presentation. MS has an onset typically occurring in young to mid adulthood, often leading to many years spent caregiving. The unpredictability of symptoms and earlier onset of disease present unique challenges for caregivers of PwMS as compared to other chronic diseases (Buhse, 2008) and significantly negatively impact caregiver quality of life (Aronson, 1997). Especially for caregivers of PwMS with advanced disease, caregiving activities can be physically and emotionally demanding and time-consuming, a combination that can be detrimental to caregivers' focus on their own care (Buchanan \& Huang, 2011; Rivera-Navarro, Morales-Gonzales, BenitoLeon, \& GEDMA, 2003). Increased caregiving demands and related stress have been associated with poorer self-care practices, poorer immune system functioning, and increased risk for physical illness among the caregivers (Long et al., 2004; Vitaliano, Zhang, \& Scanlan, 2003), including MS caregivers specifically (Forbes, While, \& Mathes, 2007; Rivera-Navarro et al., 2003). Thus, the effects of caregiver burden on health behaviors and ensuing health outcomes can be understood within the framework of a Stress/Health Model, which was formulated based upon psychological and physical effects of caregiving stress observed among caregivers of persons with dementia (Schulz, Visintainer, \& Williamson, 1990).

The Stress/Health Model proposes that stressors are met with threatening appraisals of stress (e.g., related to care recipient impairment, time spent caregiving, role stress), which in turn impact caregiver perceived stress, emotional and behavioral responses to stress, and finally, through the cumulative effects of caregiver burden, affect physical and mental health endpoints. Interventions for improving the health and well-being of dementia caregivers using the Stress/Health framework attempt to ameliorate this negative impact by focusing on caregivers' emotional and behavioral responses to stressors, for example by providing information and support to improve self-care practices (Schulz \& Martire, 2004), including enhancing caregivers' stress control and supportive communication from social contacts (Roscoe, Corsentino, Watkins, McCall, \& Sanchez-Ramos, 2009).

\section{Promoting stress management in caregivers}

Caregivers who engage in health-promoting behaviors (e.g., limiting alcohol use, exercising regularly, eating a balanced diet, getting enough sleep, and utilizing social supports) can prevent or decrease the likelihood of developing illnesses via direct and indirect effects of these behaviors while also managing the stress of their caregiving duties (Segrin \& Passalacqua, 2010; Smith \& Pergola, 2006). Despite the health benefits of effective stress management, caregivers of PwMS may struggle to find ways to balance their own needs with the care recipient's needs, calling for ongoing attention to caregiver health practices from clinicians (O'Brien, 1993).

Health professionals have been identified as important vehicles for the communication of health promotion information (Marks, Allegrante, \& Lorig, 2005; McKeown, PorterArmstrong, \& Baxter, 2003) and support for caregivers during stressful times. For example, caregivers receiving health promotion counseling from care recipients' home health nurses were able to improve their engagement in various health behaviors 
(e.g., physical activity, maintaining a healthy diet, managing stress) (Chen, 1999). However, some research has indicated that physicians do not necessarily identify discussions about caregiver needs as part of their role (Yaffe, Orzeck, \& Barylak, 2002).

Given MS health care professionals' specialized knowledge of the demands of MS and the unique challenges associated with symptom presentation and disease course, they may be preferable sources of health promotion information for MS caregivers compared to family or friends (McKeown, PorterArmstrong, \& Baxter, 2004). Further, because MS caregivers may interact with their care recipients' health care provider more often than they interact with their own physicians (Burton, Newsom, Schulz, Hirsch, \& German, 1997), MS providers also may have more opportunities to recognize caregiver burden early and direct caregivers to appropriate resources or information (Buhse, 2008). Within the context of MS care recipients' medical appointments, providers can involve caregivers and emphasize the importance of self-care and health-promoting behaviors, affording intervention to caregivers in familiar settings, at convenient times, and through extrafamilial social supports (McKeown et al., 2003; Ostwald, 2009). Although there is potential for health promotion to occur via health professionals, these supports may not always be easily accessible (Finlayson, Dahl Garcia, \& Preissner, 2008) or may not be used consistently when they are available (McKeown et al., 2004). MS caregivers have reported greater dissatisfaction with the quality of support services they receive for their roles as compared to caregivers of individuals with other neurological diseases but also reported positive benefits of support and information provided by health care professionals (Corry \& While, 2009). This is notable as perceptions of supportive communication are more strongly related to caregiver well-being than are objective measures of support (e.g., frequency; Roscoe et al., 2009). For application and intervention specific to caregiver health, additional research is needed that identifies the essential content of health promotion communication, effective methods for information delivery, and caregiver, care recipient, and provider characteristics influential to receiving health promotion messages and implementing related health behaviors.

\section{Hypotheses}

The Stress/Health Model has provided a solid theoretical basis for understanding dementia caregivers' distress and has been cited broadly in dementia caregiving research (particularly of the Alzheimer's type) within the context of designing and evaluating interventions that aim to reduce caregiver burden and improve caregiver health and quality of life (for a review, see Schulz, Martire, \& Klinger, 2005). However, this model has not been applied in understanding the stressors of MS caregiving despite similarities in disease burden (e.g., cognitive impairment, mood dysregulation); there also are important differences in MS caregiving (i.e., more variable course of disease, more years spent caregiving) that may uniquely affect MS caregivers and require different approaches to managing stress and promoting health. Building upon work outside of
MS that has indicated the stress management benefits of health-promoting behaviors (Acton, 2002; Schulz \& Martire, 2004; Smith \& Pergola, 2006) and the potential utility of health providers as critical sources of support for self-care (Finlayson et al., 2008; Marks et al., 2005), the present study sought to test a cohesive model of these factors in predicting stress management behaviors among MS caregivers. Using the Stress/Health Model as guidance, we tested whether engagement in health-promoting behaviors and perceived provider communication encouraging these behaviors would be associated with caregivers' stress control (e.g., the emotional/behavioral response to stress). Specifically, our hypotheses were as follows:

H1: Increased caregiver distress would be associated with poorer engagement in stress management behaviors (i.e., testing the perceived stress-emotional/behavioral response link of the Stress/Health Model).

$\mathrm{H} 2$ : Increased engagement in health-promoting behaviors would be associated with improved caregiver stress management behaviors (testing the potential impact of self-care and preventive health practices on the emotional/behavioral response).

H3: More perceived communication with MS care recipients' physicians regarding engagement in health-promoting behaviors would be associated with increased caregiver stress management behaviors (testing the relationship between health promotion communication and emotional/behavioral response).

\section{Method}

\section{Participants}

The survey study consisted of 67 adult caregivers of PwMS recruited from a multiple sclerosis clinic at an academic medical center in the southeastern United States. To be eligible for the study, the MS caregiver needed to be an informal caregiver (i.e., family, friend), provide at least 1 hour of assistance per week to the care recipient, and be at least 18 years of age. Most caregivers were spouses (59.7\%) and had a mean length of relationship with the care recipient of 28 years $(S D=14.7)$. Caregiver mean age was 51.8 years $(S D=11.4)$. A majority of the participants were female (53.7\%), and most identified as Caucasian (56.7\%). The years spent caregiving ranged from 1 to $38(M=8.7$, $S D=7.8$ ). Most caregivers (52.3\%) performed 11 or more hours of caregiving tasks per week (mean hours spent caregiving per week $=17.5, S D=20.77$ ). The care recipient's disability level as rated by the caregiver was in the moderate range for most of the sample $(65.7 \%)$.

\section{Measures}

\section{Demographic survey}

Participants were asked to complete a demographic survey consisting of questions regarding the characteristics of the caregiver (e.g., age, education, ethnicity, relationship to care recipient, years of caregiving, etc.) and care recipient (e.g., years since diagnoses, level of disability). 


\section{Caregiver distress scale (CDS)}

The CDS (Cousins, Davies, Turnbull, \& Playfer, 2002) was used to assess caregivers' stress and is composed of 17 items and five subscales, including (a) the Relationship Distress Scale (RDS) (e.g., "My relationship with the person with MS depresses me"), (b) the Emotional Burden Scale (EBS) (e.g., "I feel the person with MS can only depend on me"), (c) the Care-Receiver Demands Scale (CRDS) (e.g., "The person with MS makes more requests than necessary"), (d) the Social Impact Scale (SIS) (e.g., "I take part in other social activities less"), and (e) the Personal Cost Scale (PCS) (e.g., "I feel that my own health has suffered because of the person with MS"). These scales parallel the sources of caregiver stress identified in the Stress/Health Model. The scale initially was developed for use with caregivers of persons with Parkinson's disease and used a nonspecific prompt ("With respect to your current situation as caregiver for —_ please indicate whether you personally disagree or agree with the following statements ..."). The questions were adapted in the current study to refer specifically to the PwMS as the care recipient, as illustrated by the sample items. Items are rated on a 5-point Likert-type scale from 0 (strongly disagree) to 4 (strongly agree). Alphas in the present study ranged from .66 to .94 .

\section{Health and human services healthstyle self-test (HSST)}

Participants were asked to complete the Health and Human Services HealthStyle Self-Test (HSST) (U.S. Department of Health and Human Services, 1981), a 24-item measure developed to assess various behaviors associated with good health. The scale is composed of six subscales: Cigarette Smoking, Alcohol and Drugs, Eating Habits, Exercise and Fitness, Safety, and Stress Control (the dependent variable). The HSST items are rated using a 3-point scale from 0 (almost never) to 2 (almost always), with higher scores indicating more positive health care practices. Alphas in the present study ranged from .54 to .94 .

\section{Health promotion communication scale (HPCS)}

The authors adapted and modified the Short Healthstyle Index (SHS; Bertera, 2002) to be used for caregiver assessment of perceived physician communication about health-promoting behaviors. The SHS is composed of 13 items assessing engagement in health-promoting behaviors and was derived from the HSST for use with older adults of lower socioeconomic status. Cronbach's alpha for the original scale was reported at .72 (Bertera, 2002). We selected this measure for its brevity, given that the 24-item HSST was believed to be far more specific than most MS physicians would be in communicating to caregivers about health-promoting behaviors. The SHS was adapted for use in the current study by asking caregivers to consider their interactions with a care recipient's physician about each health behavior (e.g., "When at the doctor's office accompanying the patient with MS, his/her physician encourages me to have a healthy diet," "... drink no more than 1 or 2 alcoholic beverages a day," "... relax and express my feelings freely to others," etc.). Seven items came directly from the SHS. Several other SHS items were condensed into single items because the content remained more specific than we believed would occur in health promotion communication between a care recipient's physician and the caregiver. For example, four items related to specific dietary behaviors were condensed into "have a healthy diet." Based on our clinical experiences with caregivers and the caregiver health literature (e.g., Acton, 2002; Burton et al., 1997), we also added two items related to getting adequate sleep and caring for one's emotional health. The final 11 items are rated on a 4-point scale from 0 (never) to 3 (almost always), and scores are summed such that higher scores indicate greater perceived physician encouragement of health promotion behaviors for caregivers. Internal consistency of the items was excellent with a Cronbach alpha of .94 .

\section{Procedure}

Caregivers were recruited during the care recipients' visit to the MS Center and also through the MS support group and educational programs sponsored locally by the MS Center. Participation was voluntary, and caregivers received no remuneration. Participants gave informed consent and were given the opportunity to complete the questionnaire at the time of the visit or to return it through the mail. Approval for this study was granted by the institutional review board of the medical school associated with the MS Center.

\section{Data analysis}

Study variables were selected based upon the health promotion and caregiver stress management literature cited herein. Correlations among study measures were examined to determine which would be entered into a hierarchical linear regression with caregiver stress management set as the outcome variable. Demographic variables also were examined using correlations (for continuous variables) and analysis of variance (ANOVA) or $t$-tests (for categorical variables) to determine relevance in the model. Following selection of appropriate variables based on correlations, an evidencebased approach to entry of variables into the model was undertaken, such that variables that were deemed more likely to impact stress management based on the literature were entered earlier in the hierarchical linear regression (i.e., caregiver distress [CDS] in Step 1 and engagement in healthpromoting behaviors [HSST] in Step 2); physician communication regarding health behaviors (HPCS) was entered in Step 3 of the model, given that it is a new aspect of promoting stress-management behaviors being tested. All analyses were conducted using IBM SPSS version 22. Alpha level of .05 was set for all analyses.

\section{Results}

\section{Preliminary analyses}

Pearson correlation coefficients among study variables are presented in Table 1. Regarding caregiver distress, Care Recipient Demands and Emotional Burden were related to engagement in stress management behaviors; thus, these two subscales of the CDS were selected for inclusion in the regression. Caregiver distress scores were inversely related to HPCS 
Table 1. Correlations among study measures.

\begin{tabular}{|c|c|c|c|c|c|c|c|c|c|c|c|c|}
\hline \multicolumn{13}{|c|}{ Caregiver distress scale health style self-test HPCS } \\
\hline & $\begin{array}{l}\text { 1. Emo. } \\
\text { burden }\end{array}$ & $\begin{array}{l}\text { 2. Soc. } \\
\text { impact }\end{array}$ & $\begin{array}{l}\text { 3. Pers. } \\
\text { cost }\end{array}$ & $\begin{array}{l}\text { 4. Recip. } \\
\text { demands }\end{array}$ & $\begin{array}{l}\text { 5. Rel. } \\
\text { distress }\end{array}$ & $\begin{array}{c}6 . \\
\text { Smoking }\end{array}$ & $\begin{array}{c}7 . \\
\text { Alcohol }\end{array}$ & 8. Diet & 9. PA & $\begin{array}{c}\text { 10. Stress } \\
\text { control }\end{array}$ & $\begin{array}{c}11 . \\
\text { Safety }\end{array}$ & \\
\hline 1. & & $.36^{* *}$ & $.70^{* *}$ & $.81^{* *}$ & $.72^{* *}$ & .03 & .01 & -.16 & -.13 & $-.38^{* *}$ & $-.35^{* *}$ & -.18 \\
\hline 3. & & & & $.73^{* *}$ & $.62^{* *}$ & .05 & .06 & $-.32^{*}$ & -.23 & -.23 & -.18 & $-.26^{*}$ \\
\hline 4. & & & & & $.67^{* *}$ & .01 & .09 & $-.40^{* *}$ & $-.29^{*}$ & $-.36^{* *}$ & -.20 & $-.36^{* *}$ \\
\hline 5. & & & & & & -.07 & .04 & -.15 & -.07 & -.21 & -.25 & -.11 \\
\hline 6. & & & & & & & .24 & .20 & .18 & $.27^{*}$ & .10 & -.07 \\
\hline 9. & & & & & & & & & & $.33^{*}$ & -.02 & .25 \\
\hline 10. & & & & & & & & & & & .14 & $.37^{* *}$ \\
\hline 11. & & & & & & & & & & & & -.17 \\
\hline
\end{tabular}

${ }^{*} p<.05,{ }^{* *} p<.001$.

and HSST scores, indicating greater perceived encouragement for and engagement in health-promoting behaviors at lower levels of distress. HPCS and stress management scores were positively correlated, suggesting greater engagement in stress control with greater perceived encouragement of health behaviors. Four of the health-promoting behaviors (i.e., smoking, alcohol use, diet, and exercise) were related to stress management (indicating an association between engaging in health-promoting behaviors and managing stress) and were entered into the regression.

None of the participant demographics were related to the outcome variable (stress management), and thus they were not included in the regression. However, several of the caregiver demographics were related to the independent variables (including physician communication) and, while not included in the final model, provide additional context regarding this variable. Specifically, communication with the physician about health behaviors was inversely related to years spent caregiving $(r=-.36, p<.01)$ and years since the care recipient was diagnosed with MS $(r=-.38, p<.01)$, such that caregivers with fewer years in that role reported more encouragement from physicians for health-promoting behaviors, and positively correlated with hours spent caregiving $(r=.72$, $p<.05)$, such that a greater number of hours spent caregiving was associated with more perceived encouragement for health behaviors.

\section{Influences on caregivers' ability to manage stress}

To test the hypothesis that caregiver engagement in stress management is associated with caregiver distress (CDS), engagement in health-promoting behaviors (HSST), and physician communication about health behaviors (HPCS), we used a hierarchical linear regression. Caregiver distress (i.e., care recipient demands and emotional burden) was associated with stress management, $F(2,52)=4.20, p=.02, R^{2}=.14$. Both subscales of the CDS were inversely related to caregiver stress management behaviors, though neither variable was significantly associated with stress management individually (see Table 2 for standardized betas). Entering engagement in health-promoting behaviors in Step 2 significantly improved the model, $F(6,48)=6.21, p=.000, R^{2}=.44$. Lower emotional burden (CDS) and better control of alcohol and drug use
Table 2. Hierarchical regression predicting stress control.

\begin{tabular}{|c|c|c|c|c|}
\hline Variables & $R^{2}$ & $F$ & $\beta$ & $t$ \\
\hline Step 1 & .14 & $4.20^{*}$ & & \\
\hline $\begin{array}{l}\text { Emotional } \\
\text { Burden }\end{array}$ & & & -.26 & -1.23 \\
\hline $\begin{array}{l}\text { Receiver } \\
\text { Demands }\end{array}$ & & & -.13 & -.64 \\
\hline Step 2 & $.44\left(\Delta R^{2}=.30^{*}\right)$ & $6.21^{*}$ & & \\
\hline $\begin{array}{l}\text { Emotional } \\
\text { Burden** }\end{array}$ & & & -.38 & -2.03 \\
\hline $\begin{array}{l}\text { Receiver } \\
\text { Demands }\end{array}$ & & & .07 & .33 \\
\hline Smoking & & & .12 & 1.08 \\
\hline Alcohol & & & .31 .12 & 2.671 .95 \\
\hline Control** & & & .09 & \\
\hline $\begin{array}{l}\text { Diet } \\
\text { Exercise }\end{array}$ & & & & 1.13 \\
\hline Step 3 & $.47\left(\Delta R^{2}=.03\right)$ & $5.95^{*}$ & & \\
\hline $\begin{array}{l}\text { Emotional } \\
\text { Burden** }\end{array}$ & & & -.41 & -2.22 \\
\hline $\begin{array}{l}\text { Demands } \\
\text { Smoking }\end{array}$ & & & .16 & 1.37 \\
\hline Alcohol & & & .28 & 2.35 \\
\hline Control $^{* *}$ & & & & \\
\hline Diet & & & .25 & 1.82 \\
\hline Exercise & & & .11 & .94 \\
\hline HPCS & & & .21 & 1.71 \\
\hline
\end{tabular}

${ }^{*} p<.01,{ }^{* *} p<.05$.

(HSST) were most closely associated with stress management. Finally, physician communication regarding health behaviors was entered into the model. The overall model remained significant and an additional amount of variance in stress management was explained, such that nearly half of the variance in stress management was accounted for $(F(7$, $\left.47)=5.95, p=.000, R^{2}=.47\right)$; however, the change in $R^{2}$ was not significant $(p=.09)$, indicating that inclusion of perceived physician encouragement for health behaviors did not add significantly to the model, though it was trending toward significance. At this step, emotional burden and alcohol control remained the variables significantly associated with stress management.

\section{Discussion}

The deleterious effects of stress on health have been recognized as an important concern in the caregiving literature. However, relatively little attention has been paid to health 
promotion strategies and even less so among caregivers of PwMS. Thus, the current study sought to examine MS caregivers' perceptions of caregiver distress, health behavior engagement, and health promotion communication from physicians of MS care recipients and their relationship to reported stress control behaviors.

Our first aim was to test the association between caregiver stress, health promotion, and engagement in stress management. Our results indicated that caregivers engaged more in health-promoting and stress management behaviors at lower reported levels of distress, consistent with the Stress/Health Model and previous research indicating poorer self-care and stress management among overburdened caregivers (e.g., Buchanan \& Huang, 2011; Ostwald, 2009). Emotional burden was particularly important in understanding caregiver stress control in our study, as well as in previous studies of caregiver stress and health (Buchanan \& Huang, 2011; Corry \& While, 2009). Thus, these findings suggest that information or support related to caregiver stress management would be most helpful if it addressed the emotional burden of caregiving. For example, encouragement to seek mental health counseling or a support group may be indicated for an individual struggling with the emotional aspects of caregiving (e.g., Buchanan \& Huang, 2011).

Next, we tested the effects of engagement in health-promoting practices on caregivers' emotional and behavioral responses to stress (i.e., stress management), as indicated in stress and health promotion models (Schulz \& Martire, 2004). For caregivers who reported greater engagement in healthpromoting activities, greater engagement in stress management behaviors also was evident. Thus, the present results align with previous work (i.e., Acton, 2002) and provide at least partial support for the Stress/Health Model (i.e., that selfcare and preventive health practices are associated with attenuated stress responses).

Finally, we wanted to explore caregiver perceptions of physician encouragement of health-promoting behaviors, specifically as they relate to caregiver stress management. Several researchers have recognized the needs of caregivers and the potential for intervention by health professionals in a context that is convenient for the caregiver (e.g., Chen, 1999; Schulz \& Martire, 2004), though there also is mixed research on physicians' perceptions of the role they play in communicating with the caregiver about health promotion and stress management (e.g., Yaffe et al., 2002). Caregiver perceptions of supportive communication about stress management have been associated with caregiver well-being regardless of frequency of communication or distress level (Roscoe et al., 2009). In the present study, MS caregivers who perceived greater encouragement for health behaviors from their providers were those with lower reported levels of distress (i.e., lower personal cost and care recipient demands); thus, these results are consistent with literature indicating that a conversation focusing on caregivers' own needs is more likely to occur during less stressful times (Wanzer, Booth-Butterfield, \& Gruber, 2004). It is interesting to note that caregivers also perceived greater encouragement for health promotion at fewer years or a greater number of hours of caregiving. One difficulty with providing support to caregivers has been noted as the unpredictability of their openness to support (e.g., McKeown et al., 2004; Yaffe et al., 2002); in light of this previous research and the present study's results, it appears that further research is needed to identify the most helpful time in the disease trajectory for physicians to initiate conversations with caregivers about their own needs. Though greater perceived encouragement of a healthy lifestyle was correlated with improved diet practices and stress control, this association was not significant in the regression model for stress management. However, the addition of care recipient physician's encouragement of health behaviors was trending toward significance ( $p=.09$ in the overall change to the model), indicating that further investigation of this variable in a larger sample of caregivers would be a reasonable next step prior to the development and testing of a specific intervention. Thus, the current study offers preliminary evidence that could be substantiated by future studies addressing some of the limitations in the current study.

\section{Limitations}

The primary limitation of the present study is the cross-sectional design, which limits the conclusions that we can draw about the direction of the relationship between the variables of interest in our study. A longitudinal study with an intervention and control group and inclusion of a larger number of caregivers would offer more strength to test our hypothesis that health professionals' encouragement mediates the relationship between caregiver distress and engagement in stress management behaviors. Future research also should include examination of intervention content, frequency, and delivery mechanisms that are most beneficial to MS caregivers and feasible to health care providers. For example, content may need to address emotional health directly (Buchanan \& Huang, 2011) and is likely to be most effective if tailored to the individual needs of the health-promotion message recipient (Robinson, Turner, Levine, \& Tian, 2011) and perceived as emotionally supportive (Roscoe et al., 2009). As previously noted, timing and delivery method of the communication also should be explored. It was beyond the scope of the present study to address these particular communication issues. Further, to fully test the Stress/Health Model, measures of caregiver physical health would be important in understanding the endpoints of distress, health-promoting behaviors, health-promotion communication, and stress management.

Sampling also should be considered in participants in future studies. Given that participants in the study had attended a clinic appointment with a PwMS or another MSrelated activity, this makes them a specific and potentially biased sample, as we do not have information about caregivers who were unable to attend or not interested in attending these activities. Additional limitations are the measurement of only one specific type of stress (i.e., caregiver distress) and use of an untested measure of health promotion communication. Regarding the measurement of stress, certainly it is possible that caregivers experience stress not directly related to their caregiving role that also impacts their ability to engage in health-promoting behaviors. Although other acute events may affect caregiver's perceived 
stress in the short term, the Stress/Health Model holds that caregiving stressors are primary (Schulz \& Martire, 2004), and the chronic stress of caregiver burden is the most detrimental to physical health (Long et al., 2004; Vitaliano et al., 2003).

As available measures of health-promotion behaviors had not been tested specifically in the context of measuring physician-caregiver communication about health behaviors, the authors opted to develop a measure to suit the study's needs specifically. Although using an untested measure could introduce a limitation into the study, it also offers an opportunity to explore a new instrument with which to assess physician-caregiver communication about self-care. Given the strong internal consistency reliability, correlations with engagement in health behaviors, and trend toward significance as a predictor variable in the regression model, this study offers initial validity evidence for the use of HPCS to examine caregivers' perceptions of health-promotion messaging by health care providers. As perceptions of support have been related to caregiver well-being (Roscoe et al., 2009), perceived support for enacting health-promoting behaviors is associated with engagement in those behaviors (Chen, 1999), and health professionals have been identified as potential sources of health-promotion information (Marks et al., 2005), an instrument to measure specifically the perceived support from health professionals for managing health behaviors would be elemental in advancing the research on caregiver-physician (or patient-physician) health-promotion communication.

Overall, this study extends the stress and health model of caregiver health promotion to a unique population and provides a starting point to understand how care recipients' physicians may be able to encourage caregivers' engagement in health-promoting behaviors and stress management.

\section{Conclusion}

Supporting the stress management efforts of MS caregivers is an important task, given the number of years spent caregiving and the increasing burden that develops as the disease progresses. Caregivers are likely to spend less time in self-care activities at higher levels of distress, and particularly with greater emotional burden, with potentially negative and serious effects on their own health. Engaging in health-promoting activities (e.g., maintaining proper nutrition and exercise, limiting alcohol use) can help to manage the stress of caregiving and potentially buffer against negative health outcomes. Health care providers are a source of support that can offer information to caregivers about engaging in health-promoting behaviors, especially stress control. Further research needs to be undertaken to best determine the extent to which this may impact caregiver health behaviors and describe how to best achieve this goal.

\section{References}

Acton, G. J. (2002). Health-promoting self-care in family caregivers. Western Journal of Nursing Research, 24, 73-86. doi:10.1177/ 01939450222045716
Aronson, K. (1997). Quality of life among persons with multiple sclerosis and their caregivers. Neurology, 48, 74-80. doi:10.1212/WNL.48.1.74

Bertera, E. M. (2002). Development and validation of a short health behaviors index for use with low socioeconomic status (SES) older adults. Journal of Clinical Geropsychology, 8, 101-115. doi:10.1023/ A:1014620608897

Buchanan, R., \& Huang, C. (2011). Health-related quality of life among informal caregivers assisting people with multiple sclerosis Disability and Rehabilitation, 33, 113-121. doi:10.3109/ 09638288.2010.486467

Buhse, M. (2008). Assessment of caregiver burden in families of persons with multiple sclerosis. Journal of Neuroscience Nursing, 40, 25-31. doi:10.1097/01376517-200802000-00005

Burton, L. C., Newsom, J. T., Schulz, R., Hirsch, C. H., \& German, P. S. (1997). Preventive health behaviors among spousal caregivers. Preventive Medicine, 26, 162-169. doi:10.1006/pmed.1996.0129

Chen, M.-Y. (1999). The effectiveness of health promotion counseling to family caregivers. Public Health Nursing, 16, 125-132. doi:10.1046/ j.1525-1446.1999.00125.x

Corry, M., \& While, A. (2009). The needs of carers of people with multiple sclerosis: A literature review. Scandinavian Journal of Caring Sciences, 23, 569-588. doi:10.1111/scs.2009.23.issue-3

Cousins, R., Davies, A. D. M., Turnbull, C. J., \& Playfer, J. R. (2002). Assessing caregiving distress: A conceptual analysis and a brief scale. British Journal of Clinical Psychology, 41, 387-403. doi:10.1348/ 014466502760387506

Finlayson, M., Dahl Garcia, J., \& Preissner, K. (2008). Development of an educational programme for caregivers of people aging with multiple sclerosis. Occupational Therapy International, 15, 4-17. doi:10.1002/ (ISSN)1557-0703

Forbes, A., While, A., \& Mathes, L. (2007). Informal carer activities, carer burden, and health status in multiple sclerosis. Clinical Rehabilitation, 21, 563-575. doi:10.1177/0269215507075035

Long, C., Krisztal, E., Rabinowitz, Y., Gillispie, Z., Oportot, M., Tse, C., \& Gallagher-Thompson, D. (2004). Caregiver stress and physical health: The case for stress management therapy. Clinical Psychologist, 8, 2228. doi:10.1080/13284200410001662596

Marks, R., Allegrante, J. P., \& Lorig, K. (2005). A review and synthesis of research evidence for self-efficacy enhancing interventions for reducing chronic disability: Implications for health education practice (Part II). Health Promotion Practice, 6, 148-156. doi:10.1177/ 1524839904266792

McKeown, L. P., Porter-Armstrong, A. P., \& Baxter, G. D. (2003). The needs and experiences of caregivers of individuals with multiple sclerosis: A systematic review. Clinical Rehabilitation, 17, 234-248. doi:10.1191/0269215503cr618oa

McKeown, L. P., Porter-Armstrong, A. P., \& Baxter, G. D. (2004). Caregivers of people with multiple sclerosis: Experiences of support. Multiple Sclerosis, 10, 219-230. doi:10.1191/ $1352458504 \mathrm{~ms} 1008 \mathrm{oa}$

O’Brien, M. T. (1993). Multiple sclerosis: Health-promoting behaviors of spousal caregivers. Journal of Neuroscience Nursing, 25, 105-112. doi:10.1097/01376517-199304000-00007

Ostwald, S. K. (2009). Who is caring for the caregiver? Promoting spousal caregiver's health. Family \& Community Health, 32, S5-S14. doi:10.1097/01.FCH.0000342835.13230.a0

Rivera-Navarro, J., Morales-Gonzalez, J. M., Benito-Leon, J., \& Madrid Demyelinating Diseases Group (GEDMA). (2003). Informal caregiving in multiple sclerosis patients: Data from the madrid demyelinating disease group study. Disability and Rehabilitation, 25, 1057-1064. doi:10.1080/0963828031000137766

Robinson, J. D., Turner, J. W., Levine, B., \& Tian, Y. (2011). Expanding the walls of the health care encounter: Support and outcomes for patients online. Health Communication, 26, 125-134. doi:10.1080/ 10410236.2010.541990

Roscoe, L. A., Corsentino, E., Watkins, S., McCall, M., \& Sanchez-Ramos, J. (2009). Well-being of family caregivers of persons with late-stage Huntington's disease: Lessons in stress and coping. Health Communication, 24, 239-248. doi:10.1080/10410230902804133 
Schulz, R., \& Martire, L. M. (2004). Family caregiving of persons with dementia: Prevalence, health effects, and support strategies. American Journal of Geriatric Psychiatry, 12, 240-249. doi:10.1097/00019442200405000-00002

Schulz, R., Martire, L. M., \& Klinger, J. N. (2005). Evidence-based caregiver interventions in geriatric psychiatry. Psychiatric Clinics of North America, 28, 1007-1038. doi:10.1016/j.psc.2005.09.003

Schulz, R., Visintainer, P., \& Williamson, G. M. (1990). Psychiatric and physical morbidity effects of caregiving. Journal of Gerontology, 45, 181-191. doi:10.1093/geronj/45.5.P181

Segrin, C., \& Passalacqua, S. A. (2010). Functions of loneliness, social support, health behaviors and stress in association with poor health. Health Communication, 25, 312-322. doi:10.1080/10410231003773334

Smith, S., \& Pergola, J. (2006). Stress management: Preventing stress through lifestyle management. EDIS. Florida Cooperative Extension
Service, University of Florida. FCS2077B. Retrieved from http://ufdc. ufl.edu/IR00002211/00001

U. S. Department of Health and Human Services. (1981). Healthstyle: A self-test. Publication number (PHS) 81-50155. Washington, DC: Department of Health and Human Services.

Vitaliano, P. P., Zhang, J., \& Scanlan, J. M. (2003). Is caregiving hazardous to one's physical health? A meta-analysis. Psychological Bulletin, 129, 946-972. doi:10.1037/0033-2909.129.6.946

Wanzer, M. B., Booth-Butterfield, M., \& Gruber, K. (2004). Perceptions of health care providers' communication: Relationships between patientcentered communication and satisfaction. Health Communication, 16, 363-384. doi:10.1207/S15327027HC1603_6

Yaffe, M. J., Orzeck, P., \& Barylak, L. (2002). Family physicians' perspectives on care of dementia patients and family caregivers. Canadian Family Physician, 54, 1008-1015. 